\title{
A solution of a hoary conundrum: the origin and properties of cosmic rays
}

\author{
A. De Rújula ${ }^{a}$ \\ aTheory Division, CERN, 1211 Geneva 23, Switzerland \\ Physics Department, Boston University, USA
}

I discuss a theory of non-solar cosmic rays (CRs) based on a single type of CR source at all energies. All observed properties of CRs are predicted in terms of very simple and completely 'standard' physics. The source of CRs is extremely 'economical': it has only one parameter to be fitted to the enormous ensemble of all of the data. All other inputs are 'priors', that is theoretical or observational items of information independent of the properties of the source of CRs and chosen to lie in their pre-established ranges.

\section{Introduction}

A couple of years ago, I presented at a large cosmic-ray (CR) conference a preliminary version of the theory [1] I am to summarize here. The coffee break subsequent to my talk was attended by hundreds of participants. A prominent CR theorist, who had heard my talk, told me: 'Your theory is unacceptable. It solves the whole problem'. He paused to make a large hand gesture embracing the surrounding crowd, and added: 'What are then all these people going to do? Was he just joking? Not that I am convinced that we have the complete solution to the problem. But, I shall argue, we have $a$ solution that Ockham would have favoured, for the reasons stated in the abstract.

The standard theory posits that, up to the knee, CRs are produced by supernovae $(\mathrm{SNe})$ 2], via shocks in the interaction of their roughly spherical non-relativistic ejecta with the interstellar medium (ISM) 3. The NASA website lists almost 70000 refereed papers on 'cosmic ray(s)', most of them theoretical and 'standard'. As implied by a fraction of these papers, the standard theory has problems: it does not accelerate CRs up to the knee (e.g. 4]), the fraction of SN remnants perhaps compatible with the acceleration of nuclear CRs is insufficient to explain the CR luminosity of the Galaxy (e.g. [5]), CRs produced by SN remnants (most of which are within the 'solar circle') would diffuse outwards, generating a directional asymmetry which is not observed (e.g. 6]). CRs above the knee are mysterious.
Our theory [7] is non-standard in that CRs are accelerated at all energies by the relativistic jets ejected by SNe. It is part of a 'unified theory of high-energy astrophysics' 1, based on the 'cannonball' model [8 of the jets of accreting black holes and neutron stars, which also explains simply the properties of gamma-ray bursts (GRBs) [9], X-ray flashes (XRFs) [10], their respective afterglows (AGs) 1112, the gamma 'background' radiation [13, the CR luminosity of our Galaxy 714, and the properties of galaxy clusters harbouring 'cooling flows' 15]. Many more and less self-referring citations are given in 79].

\section{The Cannonball Model}

The 'cannon' of the CB model is analogous to the ones responsible for the ejecta of quasars and microquasars. Long-duration GRBs, for instance, are produced in ordinary core-collapse $\mathrm{SNe}$ by jets of CBs, made of ordinary-matter plasma, and travelling with high LFs, $\gamma \sim \mathcal{O}\left(10^{3}\right)$. As a consequence of the initial star's rotation, an accretion disk is produced around the newly born compact object, either by stellar material originally close to the surface of the imploding core, or by more distant stellar matter falling back after the shock's passage 816]. A CB is emitted, as in microquasars [17, when part of the accretion disk falls abruptly onto the compact object. A summary of the CB model is given in Fig. 1

Do SNe emit cannonballs? Until 2003 18, there was only one case with data good enough to 

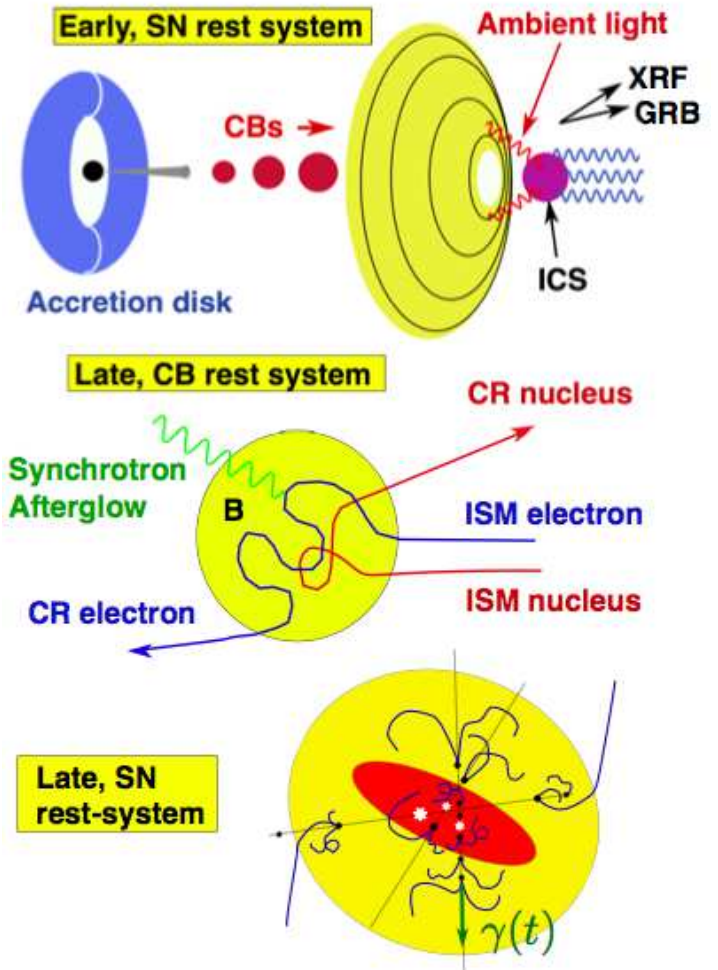

Figure 1. An 'artist's view' (not to scale) of the CB model of long-duration GRBs, XRFs and CRs. A core-collapse SN results in a compact object and a fast-rotating torus of non-ejected material. Matter (not shown) episodically accreting into the central object produces two narrowly collimated beams of CBs; only some of the 'northern' CBs are depicted. As the CBs move through the 'ambient light' surrounding the star, they forward Compton up-scatter its photons to GRB or XRF energies, depending on how close the line of sight is to the CBs' direction. Each $\mathrm{CB}$ produces a GRB 'pulse'. Later, a CB scatters ISM particles, which are isotropized by its inner magnetic field. In the SN rest system the particles are boosted by the CB's motion: they have become CRs. The synchrotron radiation of the electrons is the late AG of the GRB or XRF. The CBs generate CRs all along their trajectories, in the galaxy, its halo, and beyond, as the CBs' collisions with the ISM slow them down. CRs are also forward-produced, diffusing thereafter in the local magnetic fields. tell: SN1987A, the core-collapse SN in the LMC, whose neutrino emission was seen. Speckle interferometry data taken 30 and 38 days after the explosion [19] did show two back-to-back relativistic CBs. The approaching one was 'superluminal'.

Are GRBs made by SNe? For long-duration GRBs, the answer is affirmative [11. The first evidence for a GRB-SN association concerned SN1998bw, at redshift $z=0.0085$, observed within the angular error towards GRB 980425.

GRBs have 'afterglows': they are observable at radio to X-ray frequencies, for months after their $\gamma$-rays are seen. The optical luminosity of a 1998 bw-like SN peaks at $\sim 15(1+z)$ days. The SN light competes at that time with the AG of its GRB: it is not always detectable. It makes sense to test whether long-duration GRBs are associated with a 'standard torch' SN, akin to SN1998bw, 'transported' to their respective redshifts. The test works optimally: for all $\sim 15$ cases in which such a SN could be seen, it was seen (with varying significance) and for all $\sim 15$ cases in which the SN could not be seen, it was not seen [11. One could hardly do better. In practice SNe could not be observed at $z>1.1$.

Naturally, truly 'standard torches' do not exist, but SN1998bw made such a good job of it that we could predict 2021$]$ the SN contribution to the AG in all six recent cases of early detection of the AGs of near-by GRBs. Besides 980425-1998bw, the most convincing association was provided by the spectroscopic discovery of a $\mathrm{SN}$ in the AG of GRBs 030329 for which even the night when the SN would be discovered was foretold 21.

In a CB-model analysis of GRBs and their AGs 91112 we find that, within the pervasive cosmological factor of a few, the long-GRB-SN association would be $\sim 1: 1$. Yet, current data are insufficient to determine whether long-duration GRBs are associated with all core-collapse SNe $(\sim 70 \%$ of all SNe, including Type II) or only with Type $\mathrm{Ib} / \mathrm{c} \mathrm{SNe}(\sim 15 \%$ of core-collapse $\mathrm{SNe})$.

$C B$-model priors. The study of GRB AGs 11 12 allowed us to extract, case by case, the initial $\mathrm{LF}, \gamma_{0}$, and (less precisely) the initial mass and baryon number, $M_{0} \sim N_{\mathrm{B}} m_{p} c^{2}$, of CBs, as well as 'environmental' quantities, such as the (highly varying) ISM density and the angle between the 
jet of CBs and the observer. Typical values are:

$\gamma_{0} \equiv E /\left(M_{0} c^{2}\right) \approx\left(10^{3}\right) ; \quad N_{\mathrm{B}} \sim \mathcal{O}\left(10^{50}\right)$.

$M_{0}$ is half the mass of Mercury, i.e. tiny, compared to the mass of the parent star. The $\gamma_{0}$ values are roughly log-normally distributed around $\gamma_{0} \sim 1100$ with a width at half-maximum extending from $\gamma_{d} \sim 630$ to $\gamma_{u} \sim 1400$ [12. With these inputs, we could predict all properties of the individual $\gamma$ pulses of a GRB, each corresponding to a single CB whose electrons Compton-up-scatter the ambient light 9]. The same inputs are used to predict the properties of CRs.

While a $\mathrm{CB}$ exits from its parent $\mathrm{SN}$ and emits a GRB pulse, it is assumed [8] to be expanding, in its rest system, at a speed close to that of sound in a relativistic plasma $\left(v_{s}=c / \sqrt{3}\right)$. CBs continuously intercept electrons and nuclei of the ISM, ionized by the GRB's $\gamma$-rays. Rapidly, such an expanding CB becomes 'collisionless': its radius becomes smaller than the interaction length between the constituents of the CB and the ISM. But a CB still interacts with the charged ISM particles, for it contains a strong magnetic field.

Assume that the ISM particles entering a CB's magnetic mesh are trapped, slowly re-exiting by diffusion. Then, the CB's mass increases as:

$M_{\mathrm{CB}} \approx M_{0} \gamma_{0} /(\beta \gamma), \beta \equiv \sqrt{\gamma^{2}-1} / \gamma$

and, for an approximately hydrogenic ISM of local density $d n_{\text {in }}$, the CB's LF decreases as:

$d \gamma /\left(\beta^{3} \gamma^{3}\right) \approx-\left(m_{p} / M_{0} \gamma_{0}\right) d n_{\mathrm{in}}(\gamma)$

We approximate a $\mathrm{CB}$, in its rest system, by a sphere of radius $R(\gamma)$. The ISM particles that are intercepted - isotropized in the CB's inner magnetic mesh, and re-emitted - exert an inwards force on it that counteracts its expansion. The computed behaviour of $R(\gamma)$ is well described by:

$$
R_{\mathrm{CB}}(\gamma) \approx R_{0}\left[\gamma_{0} /(\beta \gamma)\right]^{2 / 3}, \quad R_{0} \sim 10^{14} \mathrm{~cm}
$$

The distances before a CB stops and blows up range from a fraction of a kpc to many $\mathrm{kpc}$, depending on the density profile they encounter.

The interactions of a CB and the ISM constitute the merger of two plasmas at a large relative
LF. This merger is very efficient in creating turbulent currents and magnetic fields (MFs) within the CB. We assume that the MFs, as the CB reaches a quasi-stable radius, are in 'equipartition': the MF energy density equals that of the ISM particles the CB has temporarily phagocytized. This results in a MF 11]:

$B_{\mathrm{CB}}=3 \mathrm{G}\left(\gamma / 10^{3}\right)\left[n_{\mathrm{in}} /\left(10^{-3} \mathrm{~cm}^{-3}\right)\right]^{1 / 2}$,

where $n_{\text {in }}$ is normalized to a typical value in the 'superbubbles' in which most SNe and GRBs are born. This result for $B(\gamma)$ is supported by the analysis of the spectral evolution of GRB AGs.

Charged particles interacting with turbulently moving MFs tend to gain energy: a 'Fermi' acceleration process. A 'first-principle' numerical analysis 22 of the merging of two plasmas demonstrates the generation of such chaotic MFs, and the acceleration of a small fraction of the injected particles to the approximate spectrum:

$d N / d \gamma_{A} \propto \gamma_{A}^{-2.2} \Theta\left(\gamma_{A}-\gamma\right) \Theta\left[\gamma_{\max }(\gamma)-\gamma_{A}\right]$

$\gamma_{\max }(\gamma) \simeq 10^{5} \gamma_{0}^{2 / 3}(Z / A) \gamma^{1 / 3}$

with $Z$ and $A$ the nuclear charge and mass. The first $\Theta$ function reflects the fact that it is much more likely for the light particles to gain than to lose energy in their elastic collisions with the heavy 'particles' (the CB's turbulent MF domains). The second $\Theta$ is the Larmor cutoff implied by the finite radius and $\mathrm{MF}$ of a $\mathrm{CB}$. But for the small dependence on the nuclear identity (the factor $Z / A$ ), the spectrum of Eq. (6) is universal.

The average number of pulses in a GRB's $\gamma$ rays is $\sim 6$. Thus, the total energy of the two jets of CBs emitted by a core-collapse $\mathrm{SN}$ is:

$E[$ jets $] \simeq 12 \gamma_{0} N_{\mathrm{B}} m_{p} c^{2} \simeq 2 \times 10^{51} \mathrm{erg}$.

Practically all of this energy will, in our model, be transferred to CRs and the MFs they produce.

Let $R_{\mathrm{SN}} \sim 2$ per century be the SN rate in our Galaxy. In a steady state, if the low-energy rays dominating the CR luminosity are chiefly Galactic in origin, their accelerators must compensate for the escape of CRs from the Galaxy. The Milky Way's luminosity in CRs is then:

$L_{\mathrm{CR}} \approx R_{\mathrm{SN}} E[\mathrm{jets}] \approx 1.3 \times 10^{42} \mathrm{erg} \mathrm{s}^{-1}$. 


\section{Relativistic magnetic rackets}

The essence of our theory of CRs is kinematical and trivial. In an elastic interaction of a CB at rest with ISM electrons or ions of LF $\gamma$, the light recoiling particles (of mass $m$ ) retain their incoming energy. Viewed in the ISM rest system, they have a spectrum extending, for large $\gamma$, up to $E \simeq 2 \gamma^{2} m c^{2}$. A moving $\mathrm{CB}$ is a gorgeous Lorentz-boost accelerator: the particles it elastically scatters with $\sim 100 \%$ efficiency reach up to, for $\gamma=\gamma_{0}=(1$ to 1.5$) \times 10^{3}$, an $A$-dependent knee energy $E_{\text {knee }}(A) \sim(2$ to 4$) \times 10^{15} A \mathrm{eV}$.

A particle of LF $\gamma$ entering a $\mathrm{CB}$ at rest may be accelerated by elastic interactions with the CB's turbulent plasma. Viewed in the rest system of the bulk of the CB, the interaction is inelastic and the particle may re-exit with a LF up to $\gamma_{\max } \sim$ $10^{7} \gamma$; see Eq. (4). Boosted by the CB's motion the spectrum of the scattered particles extends to $\gamma_{\mathrm{CR}} \sim 2 \times 10^{7} \gamma^{2}$, in the ultra-high-energy (UHE) domain, for $\gamma \sim \gamma_{0} \sim 10^{3}$. This powerful FermiLorentz accelerator completes our theory of CRs.

The calculation of the CR spectra takes 2.6 pages [7, which I do not have here. For $\gamma>2$, to a good approximation, the elastic contribution to the CR flux of a nucleus of ISM abundance $n_{A}$ is:

$$
\begin{aligned}
\frac{d F_{\text {elast }}}{d \gamma_{\mathrm{CR}}} & \propto n_{A}\left(\frac{A}{Z}\right)^{\beta_{\mathrm{conf}}} \int_{1}^{\gamma_{0}} \frac{d \gamma}{\gamma^{7 / 3}} G\left[\gamma, \gamma_{\mathrm{CR}}\right] \\
G\left[\gamma, \gamma_{\mathrm{CR}}\right] & \equiv \int_{\max \left[\gamma, \gamma_{\mathrm{CR}} /(2 \gamma)\right]}^{\min \left[\gamma_{0}, 2 \gamma \gamma_{\mathrm{CR}}\right]} \frac{d \gamma_{\mathrm{co}}}{\gamma_{\mathrm{Co}}^{4}}
\end{aligned}
$$

where $\beta_{\text {conf }}$ is the same 'confinement' index as in Eq. (10); $; d F_{\text {elast }} / d \gamma_{\mathrm{CR}}$ depends on the priors $n_{A}$, $\beta_{\text {conf }}$, and $\gamma_{0}$, but not on any parameter specific to the mechanism of CR acceleration. The inelastic contribution is equally simple [7.

The source spectrum of a CR nucleus is the sum of an elastic and an inelastic flux, illustrated, for protons, in Fig. 2. The elastic flux is larger than the inelastic one by a factor $f \simeq 10$ at the nominal position of the proton's knee. This ratio $f$ is the only input for which we have no 'prior' information, and the only parameter to choose in an unpredetermined range. The other parameter in Fig. 2 $N_{p}$, is the norm of the proton inelastic flux at the proton's knee. Albeit within large errors,

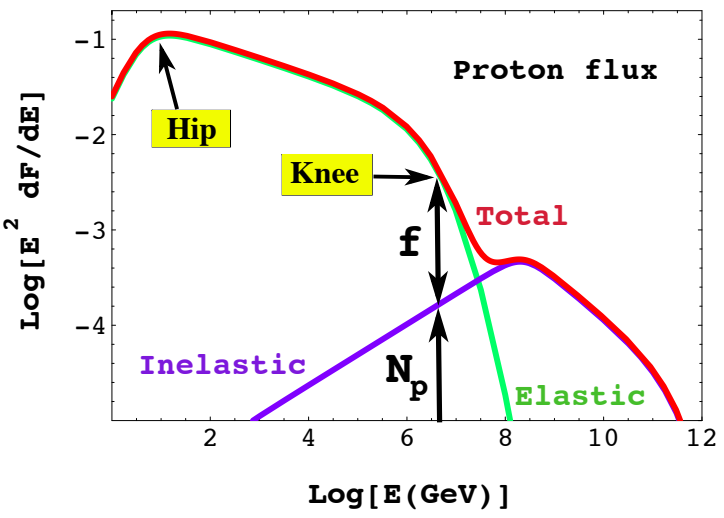

Figure 2. Contributions to the $E^{2}$-weighed proton source spectrum. Notice the predicted 'hip'.

$N_{p}$ (or $N_{p} f$ ) is determined from the luminosity of Eq. (8), and the Galactic (or universal) rate of SNe. In the domains wherein they behave as power laws, the elastic (inelastic) source spectra have indices $\beta_{\text {elast }}=13 / 6\left(\simeq \beta_{\text {elast }}+0.3\right)$.

\section{Tribulations of a Cosmic Ray}

The source and local fluxes of the main CR elements are shown in Fig. 3] These fluxes differ because, on its way from its source to the Earth, a $\mathrm{CR}$ is influenced by the ambient magnetic fields, radiation and matter. An extragalactic CR is also affected by cosmological redshift. Three types of CR 'tribulations' must be considered:

Interactions with magnetic fields. Fluxes of CRs of Galactic origin, below the free-escape (ankle) energy, $E_{\text {ankle }}(Z) \sim Z \times\left(3 \times 10^{18} \mathrm{eV}\right)$, are enhanced proportionally to their confinement time:

$\tau_{\text {conf }} \propto[Z \mathrm{GeV} /(c p)]^{\beta_{\mathrm{conf}}}, \beta_{\mathrm{conf}} \sim 0.6 \pm 0.1$.

At higher energies CRs escape or enter the Galaxy practically unhindered [23]. Lower-energy extragalactic CRs entering the Galaxy must overcome the effect of its exuding magnetic wind 7 .

Interactions with radiation, significant for CRs of extragalactic origin. The best studied one is the 'GZK' $\pi$-photoproduction on the CMB. Pair $\left(e^{+} e^{-}\right)$production is analogous. Photodissociation on the infrared CBR is also relevant.

Interactions with the ISM are well understood. CR spallation gives rise to 'secondary' CRs. 

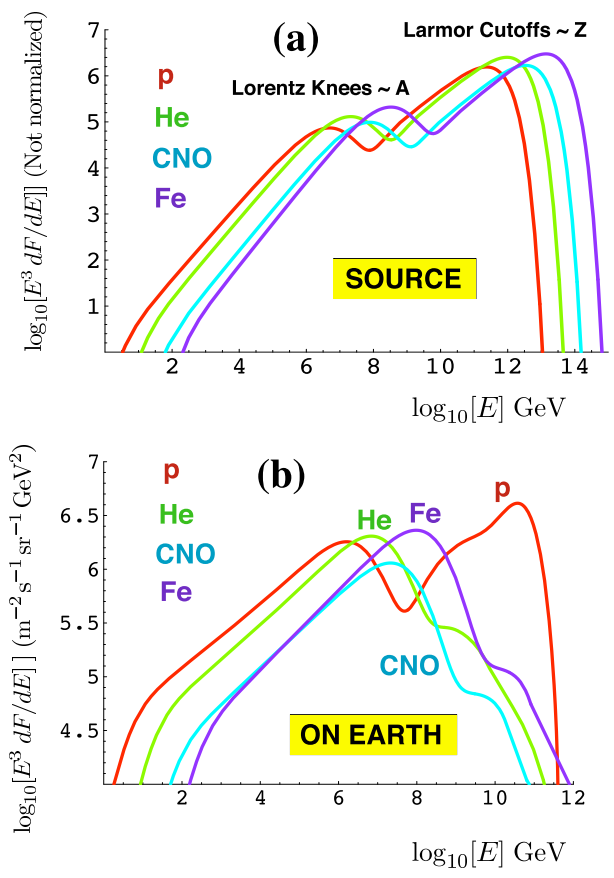

Figure 3. Predicted spectra for the abundant elements and groups. The vertical scales are $E^{3} d F / d E$. (a): The source spectra, with a common arbitrary normalization. (b): The CR spectra at the location of the Earth. Notice that both the horizontal and vertical scales are different.

\section{Results}

Relative abundances. It is customary to discuss the composition of CRs at a fixed energy $E_{A}=1 \mathrm{TeV}$. This energy is relativistic, below the corresponding knees for all $A$, and in the domain wherein the fluxes are dominantly elastic and very well approximated by a power law of in$\operatorname{dex} \beta_{\text {th }}=\beta_{\text {elast }}+\beta_{\text {conf }} \simeq 2.77$. Expressed in terms of energy $\left(E_{A} \propto A \gamma\right)$, and modified by the confinement factor of Eq. (10), Eq. (9) becomes:

$d F_{\mathrm{obs}} / d E_{A} \propto \bar{n}_{A} A^{\beta_{\mathrm{th}}-1} E_{A}^{-\beta_{\mathrm{th}}}$,

with $\bar{n}_{A}$ an average ISM abundance. At fixed energy the prediction for the CR abundances relative to hydrogen is: $X_{\mathrm{CR}}(A)=\left(\bar{n}_{A} / \bar{n}_{p}\right) A^{1.77}$. The results, for input $\bar{n}_{A}$ 's in the 'superbubbles' wherein most $\mathrm{SNe}$ occur, are shown in Fig. [4 Eq. (11) snugly reproduces the large enhancements in the heavy $\mathrm{CR}$ abundances relative to hydrogen, with respect to solar or superbubble abundances (e.g. $A^{1.77}=1242$ for $\mathrm{Fe}$ ).

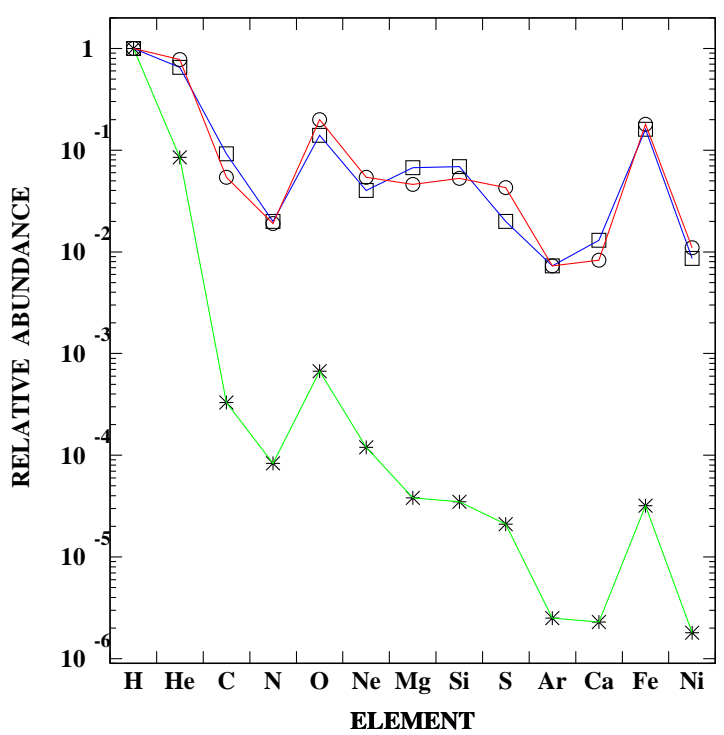

Figure 4 . The relative abundances of primary CR nuclei, from $\mathrm{H}$ to $\mathrm{Ni}$ around $1 \mathrm{TeV}$. The stars are solar-ISM abundances. The circles are the predictions, with input superbubble abundances, a bit more 'metallic' than the solar ones. The squares are the CR observations.

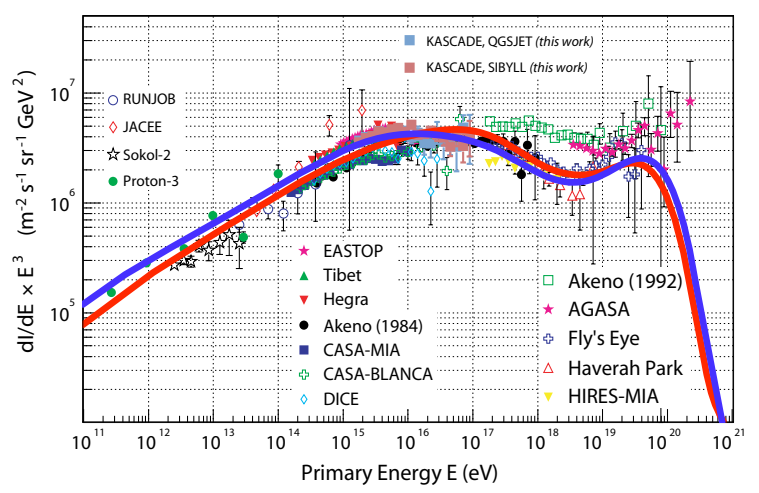

Figure 5. The all-particle CR spectrum. The vertical scale is $E^{3} d F / d E$. Some of the high-energy data disagree with others, and with our theory, which opts for the results of fluorescence detectors. The two curves correspond to two very different choices for the 'penetrability' of the Galaxy to extragalactic CRs of energy below the ankle and have slightly different values of two priors (both within errors): the width of the $\gamma_{0}$ distribution and $\beta_{\text {conf }}$, defined in Eq. (10). 
6

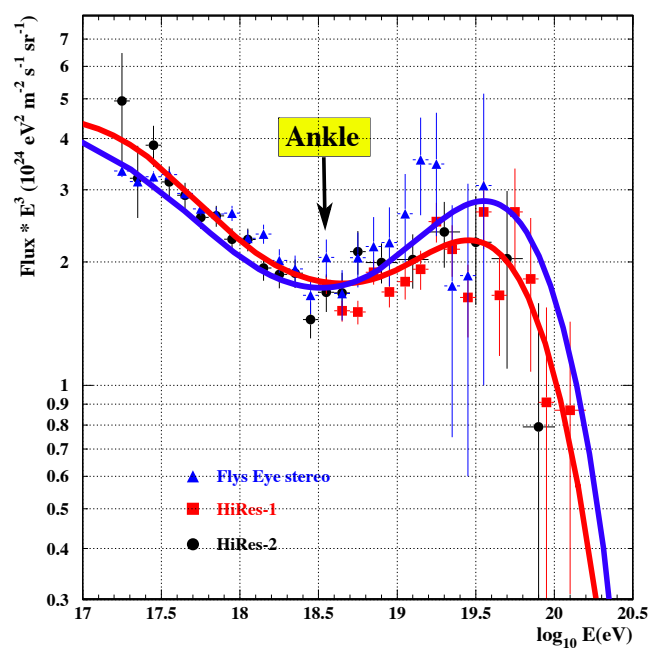

Figure 6. The $E^{3}$-weighed UHECR spectrum [24. The colour coding is as in Fig. [5]

The all-particle spectrum is shown in Fig. 5] The curves in this figure (and in later ones) correspond to two very different choices of the Galaxy's 'penetrability' to extragalactic CRs [7].

The UHECR all-particle spectrum is shown in Fig. 6] At $E=E_{\text {ankle }}$ the extragalactic contribution is $\sim 1 / 2$ of the data. The flux shape and norm, at this energy or above it, are approximate but 'absolute': they are the 'look-back-time' integral of the CR flux due to SNe in other galaxies. The shape of the high-energy end-point and the height of the hump reflect not only the GZK cutoff, but also the 'Larmor' cutoff for protons.

The knee region. There are recent KASKADE data attempting to disentangle the spectra of individual elements or groups in the knee region. Our predictions for the spectra of $\mathrm{H}, \mathrm{He}$ and $\mathrm{Fe}$ are shown in Fig. 7 The green line in the proton entry has a narrow $\gamma_{0}$ distribution, the red and blue lines have wider distributions (all within the errors in this 'prior') and correspond to the equally coloured lines in Figs. 5 and 6.

The low-energy spectra. In Fig. 8 we show the weighted spectra $E_{k}^{2.5} d F / d E_{k}$ of protons and $\alpha$ particles, as functions of $E_{k}$, the kinetic energy per nucleon. The data were taken at various times in the 11-year solar cycle. The most intense fluxes correspond to data near a solar minimum. The
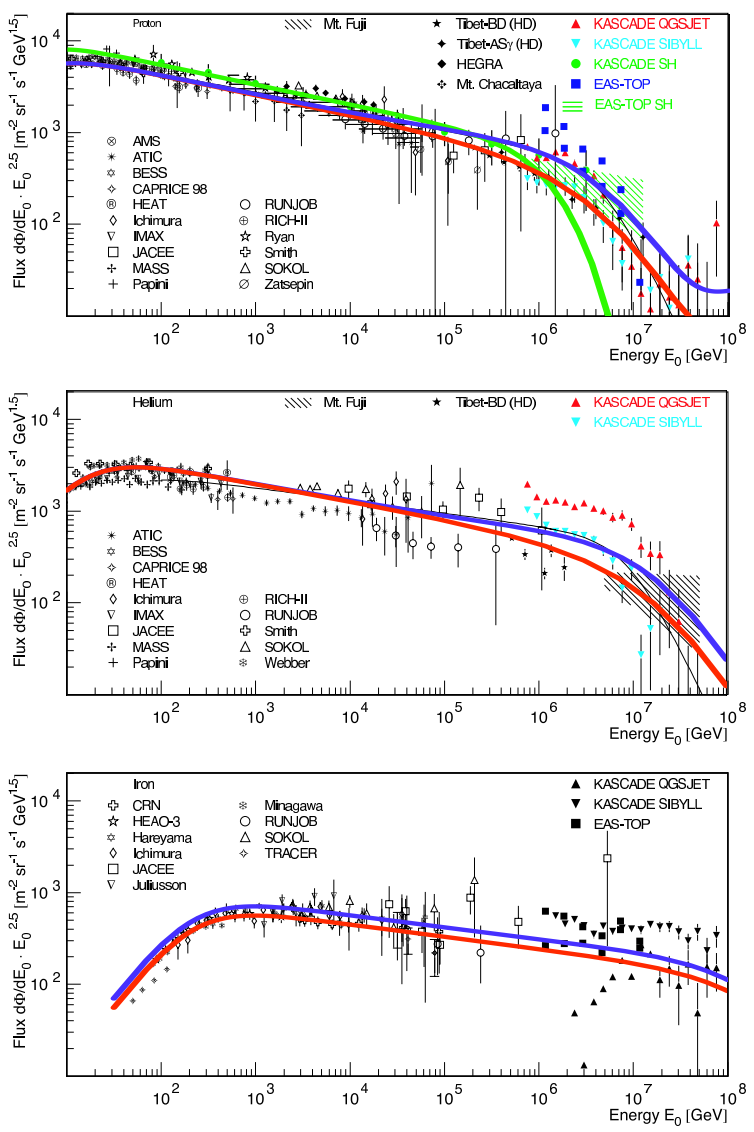

Figure 7. Measurements of individual-element CR spectra in the 'knee' region [25]. The vertical scales are $E^{2.5} d F / d E$. Top: protons; middle: $\alpha$ particles; bottom: iron nuclei. The data were kindly provided to us by K.H. Kampert. The colour coding is as in Figs. 5] and 6] The green line in the proton entry corresponds to a narrow prior $\gamma_{0}$-distribution. The $\alpha$ data and, more so, the Fe data, show the predicted low-energy 'hips', see also Figs. 2 and 8 Notice the dependence of the KASKADE results on the chosen 'shower MonteCarlo'. The data are insufficient to tell apart $E_{\text {knee }} \propto A$ (our prediction) from $E_{\text {knee }} \propto Z$ (sometimes quoted as the standard-theory result, but correct iff the limit is a Larmor cutoff and CR nuclei were accelerated up to their knees). 


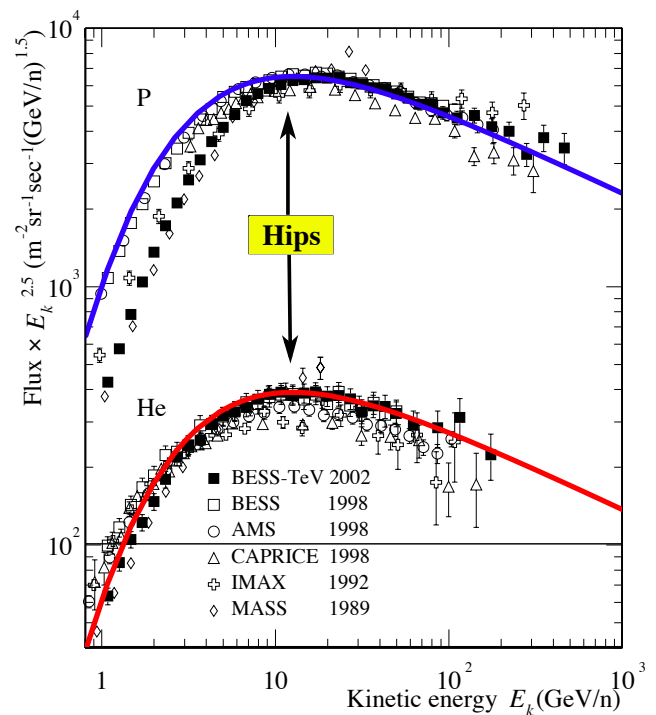

Figure 8. The very-low-energy fluxes of protons and $\alpha$ particles at various times in a solar cycle. The 1998 data are close to solar-minimum time.

curves do not model the effects of the solar wind. They should agree best with the solar-minimum data, as they do, particularly for protons. The theoretical source spectra, dominated by the elastic contribution, are given by Eq. (9). These data are well below the elastic cutoff at $\gamma_{\mathrm{CR}} \simeq 2 \gamma_{0}^{2}$, and the predictions are independent of the $\gamma_{0}$ distribution. Thus, the shape of the theoretical spectra is, in this energy domain, parameter-free.

Rough measures of CR composition. The evolution of the CR composition with energy is often presented in terms of the mean logarithmic atomic weight $\langle\ln A\rangle$, or of the depth into the atmosphere of the 'maximum' of the CR-generated shower, $X_{\max }$. The predicted $\langle\ln A(E)\rangle$ is compared with relatively low-energy data in Fig. 9 Results for $X_{\max }(E)$, are shown in Fig. 10

The predicted $\langle\ln A(E)\rangle$ at all energies, shown in Fig. 11 shows how, at very high energies, the flux is once more Fe-dominated: lighter elements have reached their GZK, acceleration and Galactic-escape cutoffs. Naturally, this prediction is sensitive to the details of Galactic escape and extragalactic photodissociation.

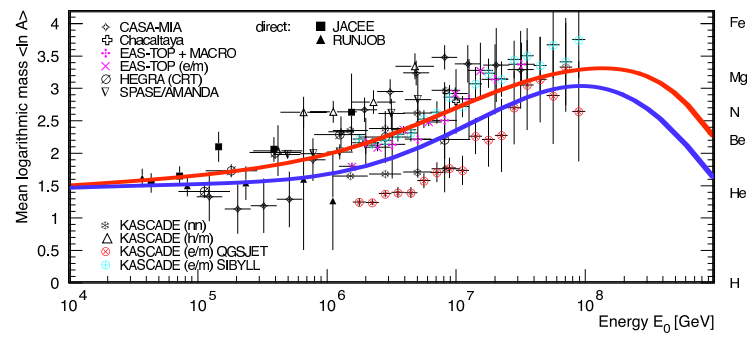

Figure 9. Mean logarithmic mass of CRs. Data points were compiled by Hoerandel [25] from experiments measuring electrons, muons, and hadrons at ground level. The colour-coded lines correspond to the same choices of priors as in Figs. 50 The compilation of data was kindly provided to us by K.H. Kampert.

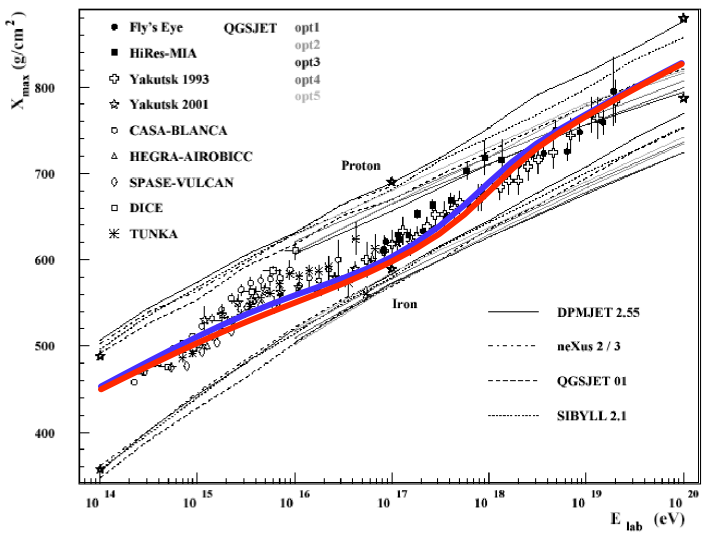

Figure 10. The depth of shower maximum as a function of energy. The data are from a compilation in Ref. [26]. The colour-coded lines correspond to the same choices as in Figs. 507 and 9 The theoretical lines are constructed with the simplified method of Wigmans [27], arguably as good as any currently-used MonteCarlo. 


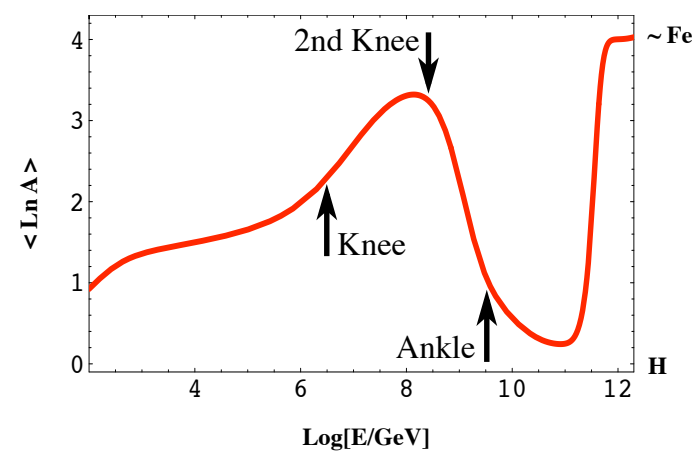

Figure 11. Predicted $\langle\ln A(E)\rangle$ at all energies.

Cosmic-ray electrons. Electrons and nuclei are accelerated by the 'magnetic-racket' CBs in the same manner [713]. The functional form of their source spectra is therefore the same, $d F_{s} / d \gamma \propto$ $\gamma^{\beta_{\text {elast }}}$, in the range $10<\gamma<10^{6}$. Electrons lose energy much more efficiently than protons in their interactions with radiation, MFs and the ISM. The rates $-d E / d t \propto E^{\alpha}$ of their various mechanisms of energy loss have different $\alpha$ 's. For Coulomb losses $\alpha=0$; for bremsstrahlung $\alpha=1$; for inverse Compton scattering (ICS) and synchrotron losses at the relevant energies, $\alpha=2$. At sufficiently high energy, the radiative energy loss dominates the others. In this domain, the steady-state solution of the equation describing the radiation-modified electron spectrum, for a source $d F_{s} / d E \propto E^{-\beta_{\text {elast }}}$, is simply 2813 :

$d F_{e} / d E \propto E^{-\beta_{e}} ; \quad \beta_{e}=\beta_{\text {elast }}+1 \approx 3.17$.

This result agrees with the observed slope of the CRE spectrum; see Fig. [12] The best-fitted value above $E \sim 6 \mathrm{GeV}$ is $\beta_{\mathrm{obs}}=3.2 \pm 0.10$, and the fit is excellent if the experiments are recalibrated to the same flux at high energy [13.

The GBR. The existence of a diffuse gamma background radiation, suggested by data from the SAS 2 satellite, was confirmed by the EGRET instrument on the Compton Gamma Ray Observatory [30]. We call 'the GBR' the diffuse emission observed by EGRET by masking the galactic plane at latitudes $|\mathrm{b}| \leq 10^{\circ}$, as well as the galactic centre at $|\mathrm{b}| \leq 30^{\circ}$ for longitudes

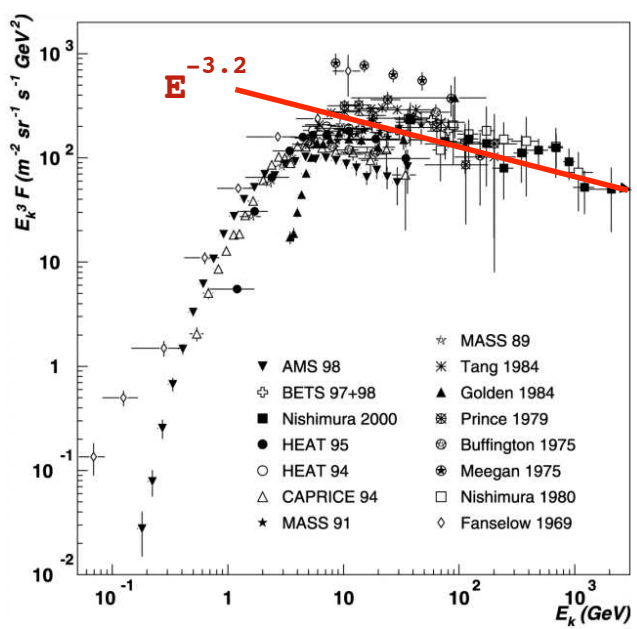

Figure 12. The CRE spectrum, compiled in [29]. The line is the central result of a power fit to the higher-energy data; its slope is $3.2 \pm 0.1$ [30]. The different slope below $\sim 6 \mathrm{GeV}$ results from the diffusion of electrons in the Galactic MF [7].

$|\mathrm{l}| \leq 40^{\circ}$, and by extrapolating to zero column density, to eliminate the $\pi^{0}$ and bremsstrahlung contributions to the observations and to tame the model-dependence of the results. Outside this 'mask', the GBR flux integrated over all directions, shown in Fig. 13, is well described by a power law $d F_{\gamma} / d E \propto E^{-2.10 \pm 0.03}$ [30].

The EGRET GBR data show a significant deviation from isotropy, correlated with the structure of the Galaxy and our position relative to its centre 28. Contrariwise, the GBR's spectral index is uncannily directionally uniform. These facts suggest a GBR that is partially local, as opposed to dominantly cosmological, and a common origin for the Galactic and extragalactic contributions.

In 2813] we have analyzed the directional and spectral properties of the EGRET data and concluded that the GBR is produced by ICS of CREs on starlight and the CBR. It has comparable contributions from CREs in a Galactic halo of dimensions akin to the hadronic-CR confinement volume (a directional and local source) and from other galaxies (an isotropic cosmological component). Thus, the GBR is a CR 'secondary'. Its spectral index is the same for the local and cos- 


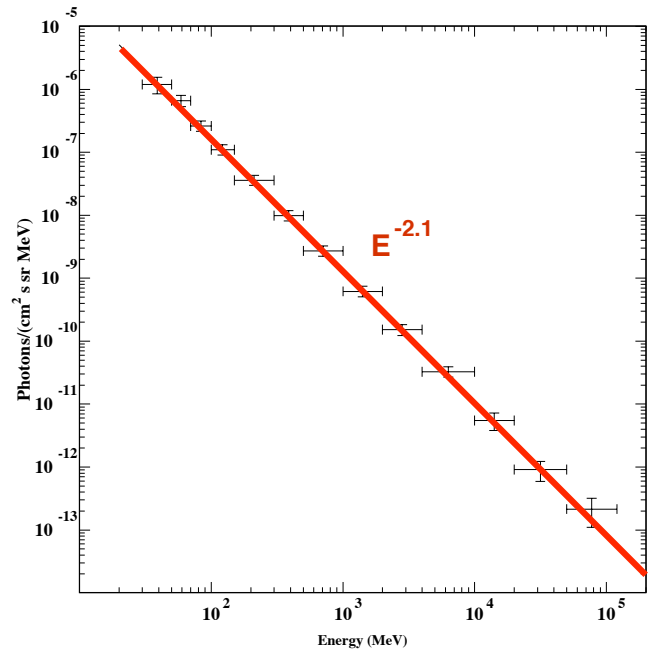

Figure 13. The GBR spectrum, measured by EGRET [30. The line is the central result of a power-law fit of slope $2.10 \pm 0.03$.

mological contributions.

If produced by ICS by electrons with the spectrum of Eq. (12), the GBR has a spectrum:

$d F_{\gamma}^{i} / d E \propto E^{-\beta_{\gamma}}, \quad \beta_{\gamma}=\left(\beta_{e}-1\right) / 2 \simeq 2.08$.

The predicted photon spectral index 28113] coincides with the measured one, $2.10 \pm 0.03$ [30].

Other predictions. Our CR theory explains other observations that I have no space to discuss here: the slight differences between the slopes of the spectra of the hadronic CRs, the deduced confinement time and volume of CR electrons and nuclei in the Galaxy 7, and the normalization and directional dependence of the GBR flux [13].

\section{Discussion and conclusions}

Our theory is incomplete in several respects. The ejection of CBs in episodes of accretion onto a compact object is supported by observations, but not fundamentally understood (this is also the case for the ejection of SN shells). Because CBs deposit CRs along their kpc-long trajectories, CR diffusion does not play a crucial role, but we have not studied it in detail (in the standard theory diffusion results in directional asymmetries that are not observed, and calls for ad-hoc remedies, such as ' $\mathrm{CR}$ reacceleration'). The temporary confinement of CRs - in the Galaxy or within a CB - is not fully understood. Neither is the 'penetrability' of the Galaxy to extragalactic CRs of energy below the ankle. We have studied the dynamics of the expansion of $\mathrm{CBs}$, but not modelled it in minute detail. We contend that a good fraction of the original energy of CRs ends up in the production of 'equipartition' MFs 31, but we cannot predict this, nor determine the effect that it may have on the CR spectral shape.

In spite of the above limitations, we have demonstrated how our simple and single accelerators - cannonballs - are effective at all observed energies. The mechanisms of CR acceleration, particularly the 'elastic' one, could hardly be simpler: a fast massive object slows down by kicking out of its way the light particles it encounters.

Most of our results are 'robust' in that - within very large brackets - they do not depend on the specific choices of parameters and priors:

- An all-particle piecewise power-law spectrum with four features: two steepenings at the knee and the second knee, a softening at the ankle, and an end-point at the roughly-coincident GZK and proton-acceleration cutoffs.

- An UHECR flux above the ankle, which is predicted - to within a factor of a few - and otherwise parameter-free.

- A composition dependence at $1 \mathrm{TeV}$ with the observed trend, so different from that of the ISM. - A very low-energy flux whose spectral shape is independent of any CB-model 'prior' parameters. - Individual-element knees that scale like $A$ and occur at the predicted energies.

- A non-trivial shape of the individual knees: an abrupt decrease in flux, followed by a spectrum steeper than that below the knee.

- An ankle with the observed shape. The dominantly Galactic-Fe flux below it and the dominantly extragalactic-proton flux above it are comparable in magnitude at the estimated escape 'ankle' energy [23] of Galactic protons.

- A composition dependence that is almost energy-independent below the knee becomes 'heavier' from the knee to the second knee, 'lighter' again above it, and finally heavier at yet unmeasured ultra-high energies. 
- An 'extended' distribution of CR sources along CB trajectories emerging from the central realms of the Galaxy, where most SN explosions occur, implying a CR flux at the Earth's location with a much smaller and less energy-dependent anisotropy than that of standard models of CRs. - Predictions for the values of a related set of observables: the CR luminosity, confinement time and volume of the Galaxy, the spectral indices of CR electrons and of the diffuse GBR.

Our results describe the observed properties of hadronic non-solar CRs very well from the lowest energies to $\sim 10^{10} \mathrm{GeV}$. Above that energy and up to the highest observed energies, $\sim 10^{11} \mathrm{GeV}$, our theory opts for the data gathered with fluorescence detectors. Overall, the energy range for which the theory is successful covers ten decades and the flux extends over three times as many.

\section{Acknowledgements}

I thank Shlomo Dado and Arnon Dar for a long and fruitful collaboration, and Nick Antoniou, Giuseppe Cocconi, Andy Cohen, Sergio Colafrancesco, Shelly Glashow and Rainer Plaga for collaboration and/or patient discussions.

\section{REFERENCES}

1. A. De Rújula, Int. J. Mod. Phys. A20, 6562 (2005) astro-ph/0411763.

2. W. Baade, \& F. Zwicky, Proc. Nat. Acad. Sci. USA 20/5, 259 (1934).

3. I. S. Shklovsky, Dokl. Akad. Nauk USSR 91, 475 (1953).

4. P.O. Lagage \& C.J. Cesarsky, Astron. Astrophys. 125, 249 (1983).

5. F. Aharonian et al., astro-ph/0510397.

6. See R. Plaga, astro-ph/0111555 for an indepth discussion and extensive reference list.

7. A. Dar \& A. De Rújula, hep-ph/0606199

8. A. Dar \& A. De Rújula, astro-ph/0008474

9. A. Dar \& A. De Rújula, Phys. Reps. 405, 203, (2004), and references therein.

10. S. Dado, A. Dar \& A. De Rújula, Astron. \& Astrophys. 422, 381 (2004).
11. S. Dado, A. Dar \& A. De Rújula, Astron. \& Astrophys. 388, 1079 (2002).

12. S. Dado, A. Dar \& A. De Rújula, Astron. \& Astrophys. 401, 243 (2003).

13. A. Dar \& A. De Rújula, Mon. Not. Roy. Astr. Soc. 323, 391 (2001); S. Dado, A. Dar \& A. De Rújula, astro-ph/0607479

14. A. Dar \& A. De Rújula, Astrophys. J. 547, L33 (2001).

15. S. Colafrancesco, A. Dar \& A. De Rújula, Astron. \& Astrophys. 413, 441 (2004).

16. A. De Rújula, Phys. Lett. B193, 514 (1987).

17. I.F. Mirabel \& L.F. Rodriguez, Annu. Rev. Astron. Astrophys. 37, 409 (1999).

18. While in the data of G.B. Taylor et al. [Astrophys. J. 609, L1 (2004)] we see $>20 \sigma$ evidence for two CBs in the AG of GRB030329 at the expected angular separation [S. Dado, A. Dar \& A. De Rújula, astro-ph/0406325 the observers dismiss this interpretation. Some CBs may be in the eyes of the beholder.

19. P. Nisenson \& C. Papaliolios, Astrophys. J. 518, L29 (1999).

20. S. Dado, A. Dar \& A. De Rújula, Astrophys. J. 572, L143 (2002); S. Dado, A. Dar \& A. De Rújula, Astron. \& Astrophys. 393, L25 (2002); S. Dado, A. Dar \& A. De Rújula, Astrophys. J. 593, 961 (2003).

21. S. Dado, A. Dar \& A. De Rújula, Astrophys. J. 594, L89 (2003).

22. J.K. Frederiksen et al., Astrophys. J. 608, L13 (2004).

23. G. Cocconi, Nuovo Cimento 3, 1433 (1956).

24. A. Zech et al., Nucl. Phys. B Proc. Suppl. 136, 34 (2004).

25. J.R. Hoerandel, astro-ph/0508014.

26. M. Zha, J. Knapp \& S. Ostapchenko, Proc. 28th Int. Cosmic Ray Conf. (Tsukuba), ICRC 2, 515 (2003).

27. R. Wigmans, Astropart. Phys. 19, 379 (2003).

28. A. Dar, A. De Rújula \& N. Antoniou, astro-ph/9901004

29. M. Sapinski et al., Proc. 29th Int. Cosmic Ray Conference, Pune, India, 101106 (2005).

30. P. Sreekumar et al., Astrophys. J. 494, 523 (1998).

31. A. Dar \& A. De Rújula, Phys. Rev. D72, $123002(2005)$. 